\title{
Autowave regimes of heat and mass transfer in the non-isothermal through-reactors
}

\author{
A. M. Brener \& L. M. Musabekova \\ State University of South Kazakhstan, Kazakhstan
}

\begin{abstract}
The paper deals with autowave processes in non-isothermal reacting diffusion systems. The mathematical model of heat and mass transfer in a tubular through-reactor has been submitted. The model consists of the diffusion-kinetic equations for two reagents under the first-order reversible reaction and heat transfer equation with allowance for a heat of reaction. Reaction rate constants for direct and reverse stages are related to temperature according to the Arrhenius law. The cases of both the adiabatic and non-adiabatic reactor have been considered. The stationary regimes and conditions of their stability have been investigated by methods of hydrodynamic stability theory and numerical experiment. As a result, the simultaneous governing transfer parameters including thermal, diffusion and kinetic characteristics of both reagents with allowance for the two reaction stages were detailed. The existence conditions for dissipate structures which can be identified as running circular cells or wave fronts have been obtained. Results of the investigations can be applied to calculating the heat and mass transfer intensity in chemical reactors and heat exchangers.
\end{abstract}

\section{Introduction}

Studies of autowave processes in chemical systems with diffusion are of great interest for scientists and engineers of different specialties now. The problem to describe such processes in every details is too complex and far from the complete solution. At the same time, the dynamics of dissipate structures has been investigated for isothermal multiphase systems sufficiently well [1-4].

But regularities of generating and propagating autowaves in non-isothermal systems with chemical reactions accompanied by heat effects have been studied 
to an insufficient extent. It is particularly correct in reference to multistage or reversible chemical reactions for which we can observe an interaction between different trends in the process evolution [5-7].

In the present work an attempt has been to prove the possibility of existence and to investigate conditions for arising dissipate structures in the simple case of the non-isothermal reversible first-order chemical reaction.

\section{Theoretical details}

Let us consider the chemical conversion which is going on in a tubular through-reactor according to the scheme of a reversible first-order reaction:

$$
X \stackrel{k_{1}}{\stackrel{k_{2}}{\longrightarrow}} Y .
$$

Here $k_{1}$ and $k_{2}$ are the reaction rate constants for direct and reverse stages.

For an adiabatic reactor the system of mass and heat transfer equations reads

$$
\begin{gathered}
\frac{\partial C_{X}}{\partial t}=D_{X} \frac{\partial^{2} C_{X}}{\partial z^{2}}+\frac{j}{S} \frac{\partial C_{X}}{\partial z}-k_{1} C_{X}+k_{2} C_{Y}, \\
\frac{\partial C_{Y}}{\partial t}=D_{Y} \frac{\partial^{2} C_{Y}}{\partial z^{2}}+\frac{j}{S} \frac{\partial C_{Y}}{\partial z}+k_{1} C_{X}-k_{2} C_{Y}, \\
\frac{\partial T}{\partial t}=\bar{\chi} \frac{\partial^{2} T}{\partial z^{2}}+\frac{j}{S} \frac{\partial T}{\partial z}+\frac{\Delta H}{\overline{\rho c}_{p}},
\end{gathered}
$$

where $C_{X}, C_{Y}$ are the concentrations of components $X$ and $Y ; C_{X 0}$ is the inlet concentration of $X ; D_{X}, D_{Y}$ are the diffusion coefficients of reagents; $t, z$ are the time and space coordinates; $j$ is the total consumption of reagents through the reactor; $T$ is the temperature; $\bar{\chi}$ is the average temperature conductivity; $\bar{\rho}$ is the average density of the reagents mixture; $\bar{c}_{p}$ is the average specific heat; $\Delta H$ is the total heat of the reaction; $S$ is the reactor crosssection surface.

We assume that $X$ is the only inlet reagent, and all parameters and properties of reagents are constant except rate coefficients. Such assumption seems to be correct by reason of a prevailing strong dependence of rate constants on temperature according to the Arrhenius law:

$$
k_{1}=k_{10} \exp \left(-E_{1} /(R T)\right)
$$




$$
k_{2}=k_{20} \exp \left(-E_{2} /(R T)\right),
$$

where $k_{10}, k_{20}$ are the standard values of the rate constants for direct and reverse stages; $E_{1}, E_{2}$ are the activating energies for direct and reverse reaction stages; $R$ is the constant of ideal gases.

The total heat of the conversion reads

$$
\Delta H=H_{1} k_{1} C_{X}+H_{2} k_{2} C_{Y},
$$

where $H_{1}$ and $H_{2}$ are the heats of direct and reverse stages.

The suitable variables are given by

$$
t, \eta=z+\frac{j}{S} t
$$

Thus instead of (2), (3), (4) we obtain:

$$
\begin{gathered}
\frac{\partial C_{X}}{\partial t}=D_{X} \frac{\partial^{2} C_{X}}{\partial \eta^{2}}-k_{1} C_{X}+k_{2} C_{Y}, \\
\frac{\partial C_{Y}}{\partial t}=D_{Y} \frac{\partial^{2} C_{Y}}{\partial \eta^{2}}+k_{1} C_{X}-k_{2} C_{Y}, \\
\frac{\partial T}{\partial t}=\bar{\chi} \frac{\partial^{2} T}{\partial \eta^{2}}+\frac{H_{1} k_{1} C_{X}+H_{2} k_{2} C_{Y}}{\overline{\rho c}_{p}} .
\end{gathered}
$$

As rank of the matrix $\left(\begin{array}{cc}-k_{1} & k_{2} \\ k_{1} & -k_{2}\end{array}\right)$ equals 1, the system of (9), (10), (11) has a non- trivial fixed points if

$$
H_{1}+H_{2}=0
$$

The condition (12) corresponds with the Hess law.

Thus we obtain a balance of fixed concentrations in the system with the ratio:

$$
\frac{C_{X 0}}{C_{Y 0}}=\frac{k_{2}}{k_{1}}=\frac{k_{20}}{k_{10}} \exp \left(-\frac{\left(E_{2}-E_{1}\right)}{R T_{0}}\right) \text {, }
$$

where $T_{0}$ is the stationary temperature which depends on initial conditions. 
184 Advanced Computational Methods in Heat Transfer IX

Linearizing the system (9)-(11) in a neighbourhood of the fixed point and using the approximation

$$
\exp \left(-\frac{E}{R T}\right) \approx \exp \left(-\frac{E}{R T_{0}}\right)\left(1+\frac{E}{R T_{0}^{2}} \theta\right)
$$

we can write the system of perturbed equations as follows:

$$
\begin{gathered}
\frac{\partial x}{\partial t}=D_{X} \frac{\partial^{2} x}{\partial \eta^{2}}-K_{1} x+K_{2} y-K_{1} \frac{E_{1} C_{X 0}}{R T_{0}^{2}} \theta+K_{2} \frac{E_{2} C_{Y 0}}{R T_{0}^{2}} \theta, \\
\frac{\partial y}{\partial t}=D_{Y} \frac{\partial^{2} y}{\partial \eta^{2}}+K_{1} x-K_{2} y+K_{1} \frac{E_{1} C_{X 0}}{R T_{0}^{2}} \theta-K_{2} \frac{E_{2} C_{Y 0}}{R T_{0}^{2}} \theta, \\
\frac{\partial \theta}{\partial t}=\bar{\chi} \frac{\partial^{2} \theta}{\partial \eta^{2}}+\frac{H\left(K_{1} x-K_{2} y\right)}{\overline{\rho c}_{p}}+\frac{H K_{1}\left(E_{1}-E_{2}\right) C_{X 0}}{\overline{\rho c}_{p} R T_{0}^{2}} \theta,
\end{gathered}
$$

where

$$
x=C_{X}-C_{X 0}, y=C_{Y}-C_{Y 0}, \theta=T-T_{0} .
$$

With allowance for (13) we obtain:

$$
\begin{gathered}
\frac{\partial x}{\partial t}=D_{X} \frac{\partial^{2} x}{\partial \eta^{2}}-K_{1} x+K_{2} y+K_{3} \theta, \\
\frac{\partial y}{\partial t}=D_{Y} \frac{\partial^{2} y}{\partial \eta^{2}}+K_{1} x-K_{2} y-K_{3} \theta . \\
\frac{\partial \theta}{\partial t}=\bar{\chi} \frac{\partial^{2} \theta}{\partial \eta^{2}}-h\left(K_{1} x-K_{2} y\right)+K_{3} h \theta .
\end{gathered}
$$

Here:

$$
\begin{gathered}
K_{1}=\exp \left(-\frac{E_{1}}{R T_{0}}\right), K_{2}=\exp \left(-\frac{E_{2}}{R T_{0}}\right), K_{3}=\frac{K_{1}\left(E_{2}-E_{1}\right) C_{X 0}}{R T_{0}^{2}}, \\
H=H_{1}=-H_{2}, h=-\frac{H}{\overline{\rho \bar{c}}_{p}} .
\end{gathered}
$$


Let us look for dissipate structures in the form of running cells [7]:

$$
\begin{aligned}
& x=\alpha_{1} \exp (\lambda t) \sin \left(\frac{m \pi}{L} \eta\right), \\
& y=\alpha_{2} \exp (\lambda t) \sin \left(\frac{m \pi}{L} \eta\right), \\
& \theta=\alpha_{3} \exp (\lambda t) \sin \left(\frac{m \pi}{L} \eta\right),
\end{aligned}
$$

where $L$ is the characteristic longitudinal scale of reactor; $\alpha_{i}$ is the amplitude of wave structures ; $m$ is the mode number, $\lambda$ is the increment.

As a result we obtain the following linear system for perturbations amplitudes:

$$
\begin{gathered}
\left(\lambda+D_{X} \frac{m^{2} \pi^{2}}{L^{2}}+K_{1}\right) \alpha_{1}-K_{2} \alpha_{2}-K_{3} \alpha_{3}=0, \\
-K_{1} \alpha_{1}+\left(\lambda+D_{Y} \frac{m^{2} \pi^{2}}{L^{2}}+K_{2}\right) \alpha_{2}+K_{3} \alpha_{3}=0, \\
h K_{1} \alpha_{1}-h K_{2} \alpha_{2}+\left(\lambda+\bar{\chi} \frac{m^{2} \pi^{2}}{L^{2}}-h K_{3}\right) \alpha_{3}=0 .
\end{gathered}
$$

The above system has non-trivial solutions if and only if

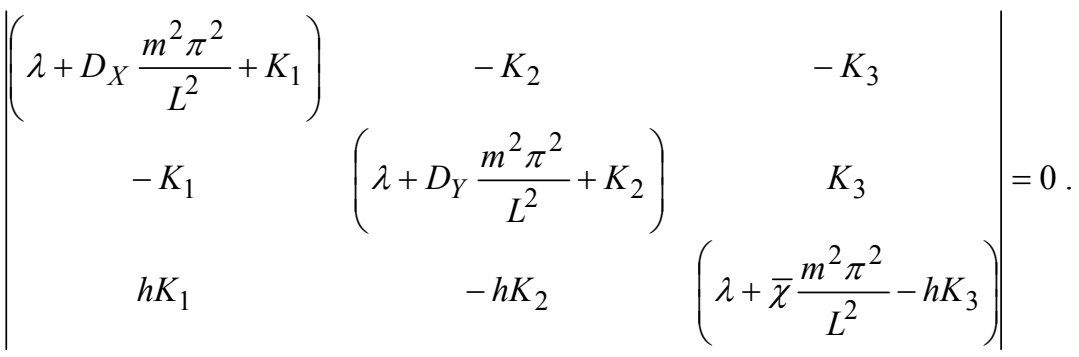

Determinant (30) can be exposed in the form:

$$
\lambda_{1} \lambda_{2} \lambda_{3}-\lambda_{1} \lambda_{2} h K_{3}+\lambda_{1} \lambda_{3} K_{2}+\lambda_{2} \lambda_{3} K_{1}=0,
$$


where

$$
\begin{aligned}
& \lambda_{1}=\lambda+D_{X} \frac{m^{2} \pi^{2}}{L^{2}}, \\
& \lambda_{2}=\lambda+D_{Y} \frac{m^{2} \pi^{2}}{L^{2}}, \\
& \lambda_{3}=\lambda+\bar{\chi} \frac{m^{2} \pi^{2}}{L^{2}} .
\end{aligned}
$$

As equation (31) is cubic concerning the increment $\lambda$ it certainly has real roots.

Therefore, existence of space dissipate structures is conditioned either by that one real root of the equation (31) equals to zero and real parts of other two roots are non-positive (case 1), or by that one real root is negative and other two roots are imaginary (case 2). Let's consider both cases.

\subsection{Case 1}

From the condition for the real root it follows:

$$
\frac{m^{2} \pi^{2}}{L^{2}}=\frac{K_{3} h}{\bar{\chi}}-\left(\frac{K_{1}}{D_{X}}+\frac{K_{2}}{D_{Y}}\right) .
$$

It is obvious that (35) can be realized only if $H_{1}<0$ (i.e. the direct reaction must be endothermic), and the following inequality is correct:

$$
\frac{K_{3} h}{\bar{\chi}}>\left(\frac{K_{1}}{D_{X}}+\frac{K_{2}}{D_{Y}}\right) .
$$

From this it follows the expression for the length of solitary wave front:

$$
\Lambda=\frac{\pi}{\sqrt{\left[\frac{K_{3} h}{\bar{\chi}}-\left(\frac{K_{1}}{D_{X}}+\frac{K_{2}}{D_{Y}}\right)\right]}} .
$$

The second condition for complex roots reduces to the system of inequalities: 


$$
\begin{gathered}
K_{1}\left(1-\frac{\bar{\chi}}{D_{X}}\right) \geq K_{2}\left(\frac{\bar{\chi}}{D_{Y}}-1\right), \\
\frac{K_{1}}{D_{X}}\left(1-\frac{\bar{\chi}}{D_{X}}\right) \geq \frac{K_{2}}{D_{Y}}\left(\frac{\bar{\chi}}{D_{Y}}-1\right) .
\end{gathered}
$$

It is easily seen that the following conditions together with (35) are sufficient for the above system correctness:

$$
\begin{gathered}
K_{1}+K_{2}>K_{3} h, \\
\left(\bar{\chi}-D_{Y}\right)\left(D_{X}-D_{Y}\right)<0 .
\end{gathered}
$$

Let's start using the dimensionless parameters:

$$
A_{1}=\frac{\bar{\chi}}{D_{X}} ; A_{2}=\frac{\bar{\chi}}{D_{Y}} ; A_{3}=\frac{K_{1}}{K_{3} h} ; A_{4}=\frac{K_{2}}{K_{3} h} .
$$

Thus, in most cases corresponding to the probability values of media properties we can display the conditions (36), (40), (41) in a graphic form. Shaded areas in the Fig.1 contain points with coordinates satisfying the system (36), (40), (41).

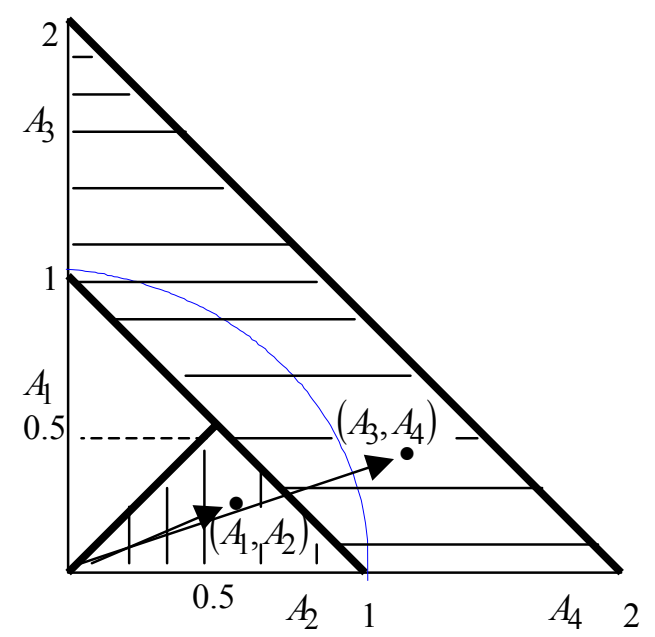

Figure 1: Domain of governing parameters (42) meeting the conditions (36), (40), (41).

As it follows from (36) the dot product between vectors $\left(A_{1}, A_{2}\right)$ and $\left(A_{3}, A_{4}\right)$ must be less than 1 . Therefore, the boundary position of the point 
( $\left.A_{3}, A_{4}\right)$ about the point $\left(A_{1}, A_{2}\right)$ is determined by inversion concerning to the circle of radius 1 in Fig. 1.

The minimum length of reactor, in which the space dissipate structures corresponding to case 1 may be generated, is given by

$$
L_{\min }=\Lambda=\frac{\pi}{\sqrt{\left[\frac{K_{3} h}{\bar{\chi}}-\left(\frac{K_{1}}{D_{X}}+\frac{K_{2}}{D_{Y}}\right)\right]}} .
$$

\subsection{Case 2}

In this case analytical investigations entail cumbersome computations. Our attempt to obtain simple sufficient conditions for existence of dissipate structures corresponding to case 2 has failed.

At the same time there are reasons to believe that under the condition (40) generation of structures corresponding to case 2 will be impossible. On the other hand it is highly probable that the following conditions will be sufficient for springing up the mentioned dissipate structures:

$$
\begin{gathered}
K_{1}+K_{2}<K_{3} h \\
h K_{3}\left(D_{X}+D_{Y}\right)<\bar{\chi}\left(K_{1}+K_{2}\right)+K_{2} D_{X}+K_{1} D_{Y} .
\end{gathered}
$$

For a non-adiabatic reactor the system of mass and heat transfer equations differs from the system (2)-(4) only in the last equation:

$$
\frac{\partial T}{\partial t}=\bar{\chi} \frac{\partial^{2} T}{\partial z^{2}}+\frac{j}{S} \frac{\partial T}{\partial z}+\frac{\Delta H}{\bar{\rho} \bar{c}_{p}}-\frac{k_{T}}{\bar{\rho} \bar{c}_{p}}\left(T-T_{m}\right),
$$

where $k_{T}$ is the ambient heat transfer coefficient and $T_{m}$ is the ambient temperature.

For existence of fixed points under the condition (12) it is necessary now to propose that

$$
T_{0}=T_{m} .
$$

Thus instead of (21) we obtain:

$$
\frac{\partial \theta}{\partial t}=\bar{\chi} \frac{\partial^{2} \theta}{\partial \eta^{2}}-h\left(K_{1} x-K_{2} y\right)+\left(K_{3} h-\frac{k_{T}}{\overline{\rho \bar{c}_{p}}}\right) \theta,
$$


It is clear that qualitative analysis of dissipate structures in a non-adiabatic through-reactor hasn't differences of principle from the case of an adiabatic reactor. However there is yet another governing parameter $k_{T} / \overline{\rho c}_{p}$.

\section{Results and discussion}

Figure 2 depicts some results of numerical tests carried out under the realistic values of physical and chemical parameters satisfying the case 1:
1) $K_{1}=3.02 \cdot 10^{-7}, K_{2}=7 \cdot 10^{-10}, K_{3} h=2.5 \cdot 10^{-7}, \frac{\bar{\chi}}{D_{X}}=0.50, \frac{\bar{\chi}}{D_{Y}}=0.67$.
2) $K_{1}=4.0 \cdot 10^{-7}, K_{2}=8.0 \cdot 10^{-10}, K_{3} h=3 \cdot 10^{-7}, \frac{\bar{\chi}}{D_{X}}=0.25, \frac{\bar{\chi}}{D_{Y}}=0.5$.
3) $K_{1}=2.0 \cdot 10^{-7}, K_{2}=1.0 \cdot 10^{-9}, K_{3} h=1.8 \cdot 10^{-7}, \frac{\bar{\chi}}{D_{X}}=0.3, \frac{\bar{\chi}}{D_{Y}}=0.6$.
4) $K_{1}=2.0 \cdot 10^{-7}, K_{2}=1.0 \cdot 10^{-9}, K_{3} h=9.5 \cdot 10^{-8}, \frac{\bar{\chi}}{D_{X}}=0.3, \frac{\bar{\chi}}{D_{Y}}=0.6$.
5) $K_{1}=2.0 \cdot 10^{-7}, K_{2}=1.0 \cdot 10^{-9}, K_{3} h=6.5 \cdot 10^{-8}, \frac{\bar{\chi}}{D_{X}}=0.3, \frac{\bar{\chi}}{D_{Y}}=0.6$.

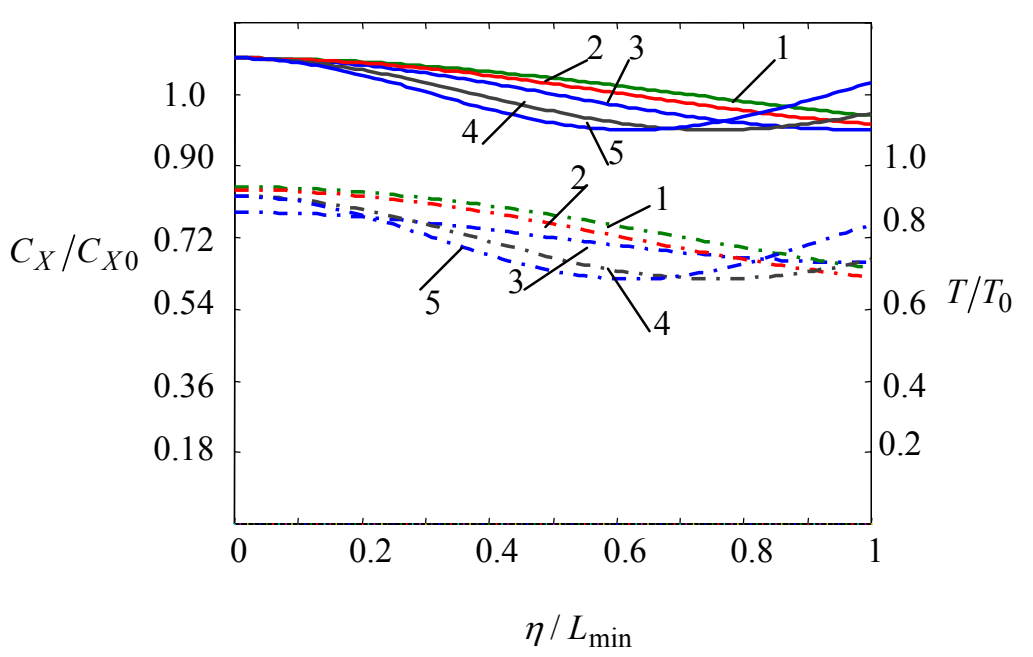

Figure 2: Heterogeneous distributions of concentration (-) and temperature $(\cdot-\cdot \cdot)$ along a tubular reactor in the case 1.

Heterogeneous distributions of concentration and temperature along a tubular reactor spring up in consequence of perturbing the stationary regime. Dissipate 
structures of this type are unstable, and they constitute an initial stage of generating running autowave fronts [6].

Dynamics of autowave structures formation and propagation in every detail can be described only by methods of non-linear stability theory $[6,7]$.

\section{Conclusion}

Formation of stable dissipate structures, such as running cells and autowaves, in non-isothermal reactors is controlled by set of diffusion and heat parameters. Our theoretical analysis allowed to detail the governing parameters which can be used for determining regimes of dissipate structures generating.

In particular, it has been clearly defined a role of rate constants dependencies on temperature with allowance for direct and reverse stages of the first-order chemical reaction. The main types of possible dissipate structures induced by these factors in a non-isothermal tubular through-reactor as well as conditions of their formation have been determined. The results of investigations are likely to be useful for calculating intensity of heat and mass transfer processes in chemical apparatuses.

\section{References}

[1] Alamgir M., Epstein I.R., Systematic Design of Chemical Oscillators. 17. Birhythmicity and Compound Oscillation in Coupled Chemical Oscillators: Chlorite-Bromate-Iodide Systems. Jour. Amer. Chem. Soc., 105, 1983, P. 2800.

[2] Ortoleva P., Ross J., Theory of Propagation of Discontinuities in Kinetic Systems with Multiple Time Scales: Fronts, Front Multiplicity and Pulses, Jour. Chem. Phys., 63, 1975, P. 3398.

[3] Schlöglm F., Chemical Reaction Models for Non-Equilibrium Phase Transitions, Z. Phys., 253, 1972, P.147.

[4] Baetens D., Van Keer R., Hosten L.H., Gas-liquid reaction: absorption accompanied by an instantaneous, irreversible reaction, Proc. Computational Modelling of Free and Moving Boundary Problems, 1997, P. 185-195.

[5] Tyson J.J., Oscillations, Bistability and Echo Waves in Models of the Belousov-Zhabotiskii Reaction., Ann. New York Acad. Sci., 316, 1979, P. 279.

[6] Showalter K., Noyes R.M., Turner H., Detailed Studies of Trigger Wave Initiation and Detection., Jour. Amer. Chem Soc., 101, 1979, P. 7463.

[7] Serdukov S.I., About existence of dissipate structures and running fronts for system of reactions with heat effect in a limited volume, Jour. of Phys. Chemistry, 59, No9, 1985, P. 2292-2296. (in Russian) 